\title{
Introduction to the identification of brain waves based on their frequency
}

\author{
Zuzana Koudelková ${ }^{1, *}$, Martin Strmiska ${ }^{1}$ \\ ${ }^{1}$ Tomas Bata University in Zlin, Faculty of Applied Informatics, Nad Stranemi 4511, 76005 Zlin, Czech Republic
}

\begin{abstract}
A Brain Computer Interface (BCI) enables to get electrical signals from the brain. In this paper, the research type of BCI was non-invasive, which capture the brain signals using electroencephalogram (EEG). EEG senses the signals from the surface of the head, where one of the important criteria is the brain wave frequency. This paper provides the measurement of EEG using the Emotiv EPOC headset and applications developed by Emotiv System. Two types of the measurements were taken to describe brain waves by their frequency. The first type of the measurements was based on logical and analytical reasoning, which was captured during solving mathematical exercise. The second type was based on relax mind during listening three types of relaxing music. The results of the measurements were displayed as a visualization of a brain activity.
\end{abstract}

\section{Introduction}

The nervous system is composed of two parts. The central nervous system (CNS) and the peripheral nervous system (PNS). The CNS consists of human brain and spinal cord. Conversely, the PNS consists of the nerves and ganglia outside the brain and spinal cord.

Human brain controls body function, such as heart activity, movement, speech, but also thinking itself, memory or emotion perception. Brain activity could be measured by the neurologic examination method electroencephalography (EEG). The principle of this method is capturing electric potential.

If the central nervous system is damaged, some body functions may be restricted. Brain computer interface systems could offer these people improved communication and independence.

Recent developments in BCI technology may see such hands-free control method realised. A BCI is a communication and control system in which the thoughts of the human mind are translated into real-world interaction without the use of the usual neural pathways and muscles. For example, users of BCI system can switch a light or change TV channels using only their imagination and without any physical movement. Recent advances in the human brain and BCI research reveal that BCI-based devices and technologies can play a significant role in the future [1-3].

The article begins with theoretical information about BCI and its types. After that, there is mentioned signal acquisition, where the brain wave distribution is described by frequency. The next topic of this article are methods and measurements. There are described device and application, which have been used. Also, there is defined using types of measurements. The following is the Results section with the measurement results, which are displayed as visualization of brain activity.

\section{Brain Computer Interface}

Brain Computer Interface acquire and analyse brain signals in real time to control external devices, communicate with others, facilitate rehabilitation or restore functions.

There are three parts of Brain Computer Interfaces. Invasive, Non-invasive and Partially invasive.

\subsection{Invasive brain computer interface}

Invasive Brain Computer Interface systems are used for the best quality signals. These electrodes are implemented into cortical issue. These types of system are used for paralyzed people, or it could be used for restoring vision by connecting the brain with external cameras. Although these BCI systems provides the best quality signals, the system is prone to scar-tissue build-up. The scar-tissue cause weak signal, which can be even lost. Because of the body reacts to a foreign object in the brain [2].

\subsection{Partially invasive brain computer interface}

Partially Invasive Brain Computer Interfaces are implanted into the skull, but outside the brain. Electrocorticographic (ECoG) uses the same technology as non-invasive electroencephalography, but the electrodes are embedded in a thin plastic pad that is placed above the cortex, beneath the dura mater. These systems produce a good signal, but weaker than Invasive BCI [2].

\footnotetext{
* Corresponding author: koudelkova@utb.cz
} 


\subsection{Non-Invasive brain computer interface}

Non-Invasive Brain Computer Interfaces means electrodes are emplaced on the surface of the skull to record changes in EEG state. The signal, which is producing has the weakest values in spite of this, the noninvasive $\mathrm{BCI}$ are the safest and easiest way to record EEG [2]. The schema of brain computer interface system is shown in Figure 1.

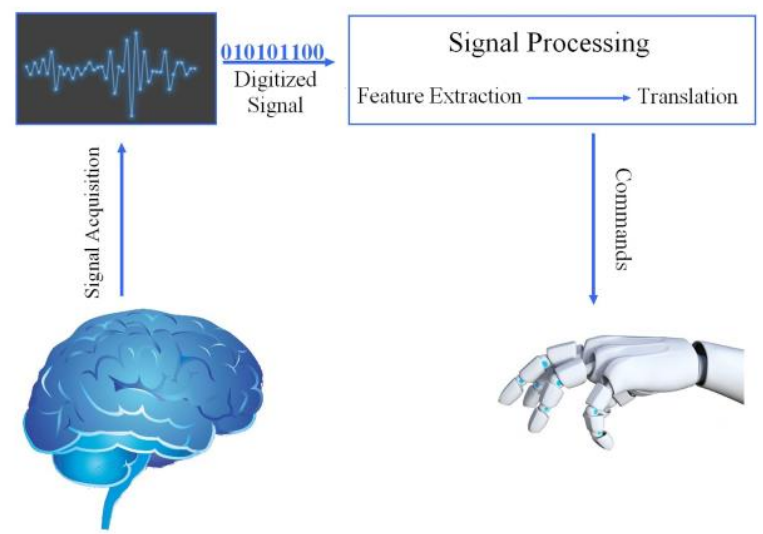

Fig. 1. Basic flow diagram of BCI system.

\section{Signal Acquisition}

EEG is the most prevalent method of signal acquisition for Brain Computer Interfaces. Many EEG systems are using International 10/20 system. This is an electrode placement strategy, which ensures ample coverage over all parts of head [4].

Brain signals are acquired by electrodes on the surface of the head. Then these signals are digitized and processed to clean and denoise data to enhance the relevant information embedded in the signals. After that, step called the feature extraction is used. It means that the brain patterns used in BCIs are characterized by certain features. Describing the signals by a few relevant values is called feature extraction. Next step is translation. This step assigns a class to a set of features extracted from the signals. This class corresponds to the type of mental states identified. Finally, the translation into a command, which means a command is associated to acquired mental state in order to control application.

The most important criteria of evaluation EEG are frequency. This is a criterion for assessing abnormalities in clinical EEG and for understanding functional behaviours in cognitive research [5].

In Table 1 there can be seen five major brain waves distinguished by their different frequency ranges (Figure 2).

Table 1. Brain wave distribution by frequency of band wave.

\begin{tabular}{|c|c|}
\hline $\begin{array}{c}\text { Name of the } \\
\text { frequency }\end{array}$ & $\begin{array}{c}\text { Frequency of band wave } \\
\mathbf{( H z )}\end{array}$ \\
\hline alpha $\alpha$ & $8-13$ \\
\hline
\end{tabular}

\begin{tabular}{|c|c|}
\hline beta $\beta$ & $13-30$ \\
\hline delta $\delta$ & $0.5-4$ \\
\hline gamma $\gamma$ & $>30$ \\
\hline theta $\theta$ & $4-8$ \\
\hline
\end{tabular}

Each brain wave has different frequency, amplitude (Figure 2) and meaning. Alpha $\alpha$ waves connect the gap between our conscious thinking and subconscious mind. It helps us to calm down or it promotes a feeling of relaxation. Beta $\beta$ waves are active in a waking state. This frequency is visible in logical-analytical reasoning. In their activity, we focus on a problem solving. Delta $\delta$ waves occur during meditation in a state of deep sleep or coma. Abnormal delta activity may occur with the person, has learning disabilities or have difficulties maintaining conscious awareness (such as in cases of brain injuries). Gamma $\gamma$ waves are important for learning, memory and information processing. Theta $\theta$ waves are involved in sleep or daydreaming. This brain wave can indicate intuition or automatic tasks [6].

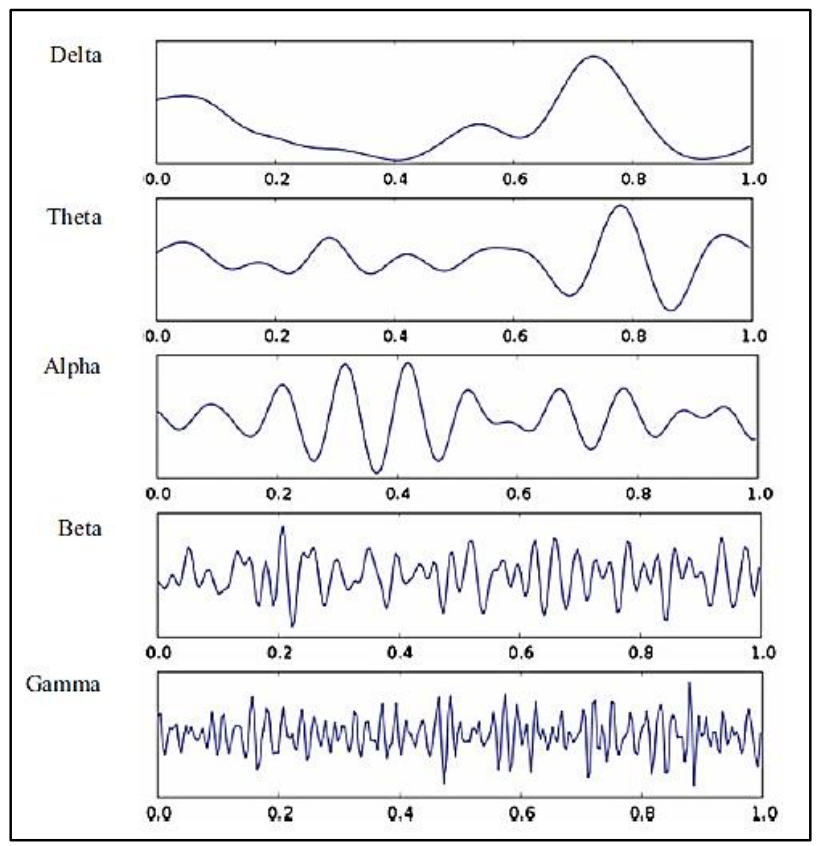

Fig. 2. Five major brain waves distinguished by their different frequency ranges [5].

\section{Methods and Measurements}

The problem of using BCI in the academic field is a high price. Best option for academic purposes is using the Emotiv EPOC device (Figure 3), which is designed and manufactured by Emotiv System. The device comprises a wireless helmet that enables the reading of feelings, emotions, and intentions of the user. The cost of this device is $799 \$$ for a Research Development Kit. 


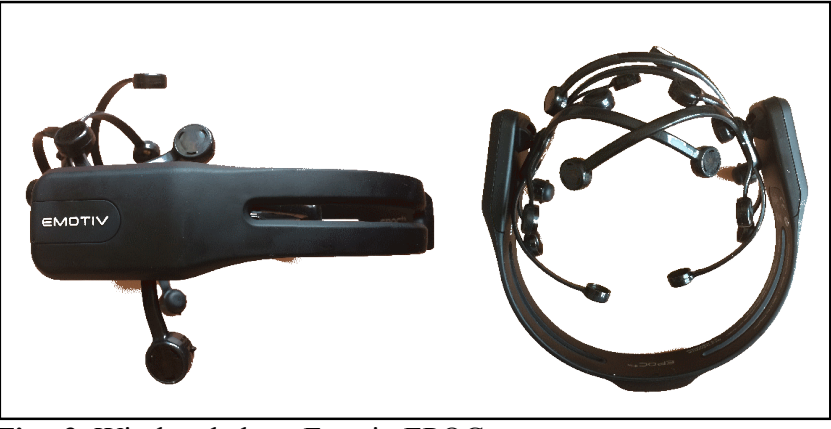

Fig. 3. Wireless helmet Emotiv EPOC.

The Emotiv EPOC device is based on International $10 / 20$ system. This headset consists of the 16 sensors on the scalp. Two of these sensors are references. The set-up of this device is fast, but there is a problem with connection of the sensors.

Measurement takes place in application Emotiv Brain Activity Map v3.3.3 (Figure 4), which is developed by Emotiv Systems. The cost of this application is $9.95 \$$. The application measure and display real-time data of four types of brain waves. Alpha, Beta, Theta and Delta. Each of these frequencies allows adjustable gain to see detailed information and relative strengths between different brain regions. Adjustable buffer size allows to see instant responses or average activity over longer periods.

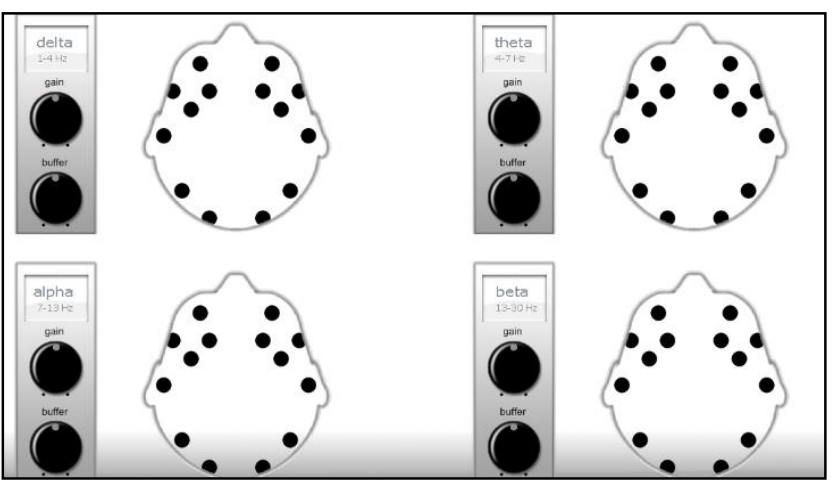

Fig. 4. Application Emotiv Brain Activity Map v3.3.3 developed by Emotiv Systems.

In this paper, there were used two types of measurement. The first type of measurement where analysis brain activity, which is produced by logical and analytical reasoning. The measurement was performed during solving mathematical exercise.

The following type of measurement was based on relaxed mind. Measurements were taken in a quiet room with a relaxing music. Three types of relaxation music were used in this paper.

\section{Results}

Results are taken as a visualization of brain activity. The colour of the weakest signal is blue. As the strength of signal increases, the colour changes to red. This is shown in Figure 5.
Fig. 5. Spectrum of signal colours from the weakest to the strongest.

\subsection{The measurement based on logical and analytical reasoning}

In the first measurement, the person used for the measurement, was given a mathematical exercise with the command to solve it.

At the beginning of the measurement it was detected that the main roles play beta and theta waves. It was proved that beta waves occur when solving a problem or they are visible at logical-analytical reasoning. It can be seen in Figure 6.

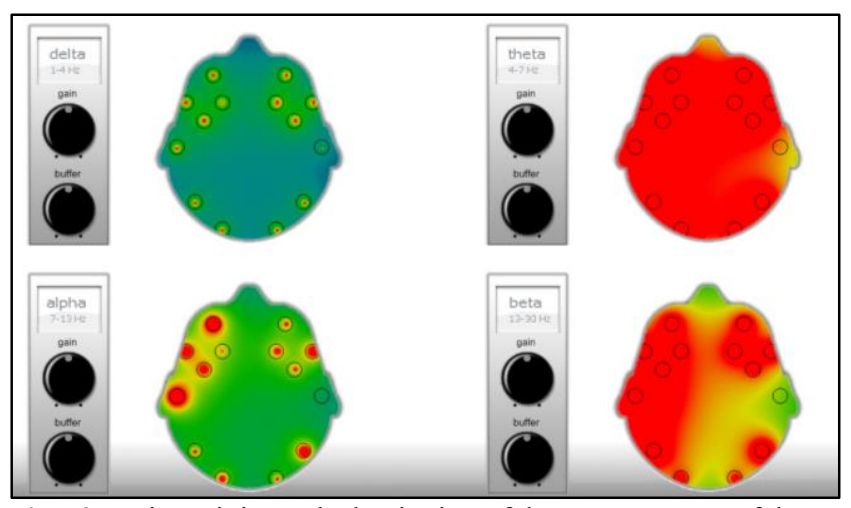

Fig. 6. Brain activity at the beginning of the measurement of the problem solving.

After one minute, the view has changed. Now, there are active only theta waves. It is because the testing person solved the exercise in the past. This was explained that theta waves occurred during the automatic problems solving. This can be seen in Figure 7.

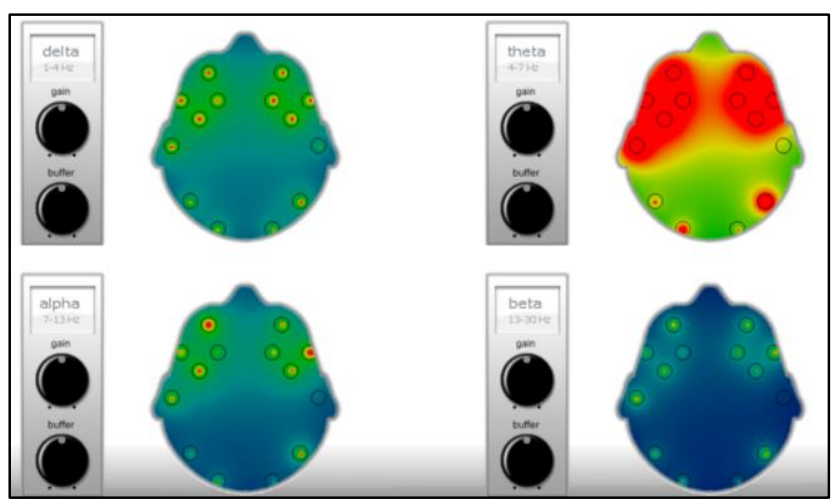

Fig. 7. Activation of theta brain waves.

\subsection{The measurement based on relaxed mind}

The second measurement was concerned with the relaxing mind. The person used for the experiment was taken a seat with a quiet room with the command to close their eyes and relax. After that, the relaxing music was played. Relaxing music should activate alpha waves. In this 
experiment, it is used three types of relaxing music. Each of that proved it. It can be seen in Figure 8 and Figure. 9.

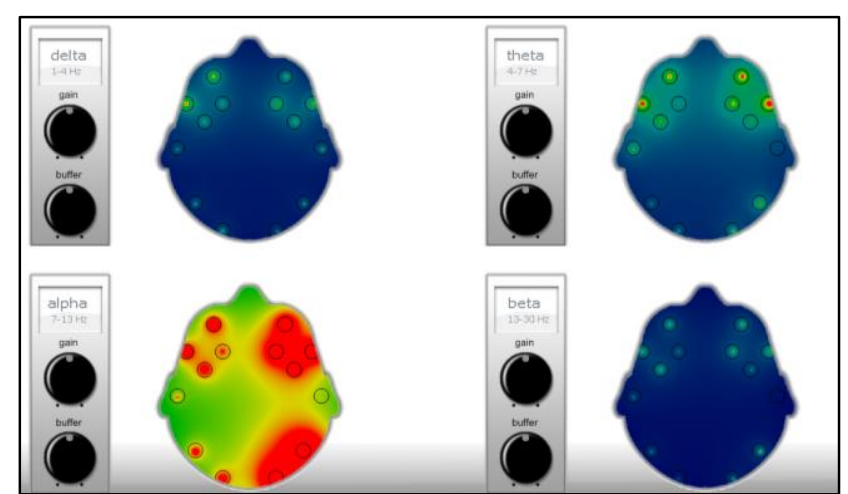

Fig. 8. Brain activity during the first type of listening relaxing music.

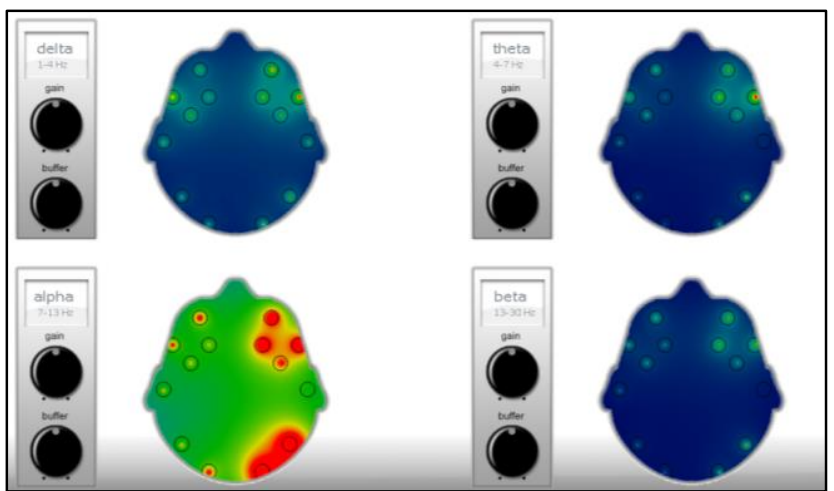

Fig. 9. Brain activity during the second type of listening relaxing music.

The last of the relaxing music also actives theta waves. Explaining the presence of theta waves is likely to be the beginning of deep relaxation. This is shown in Figure 10.

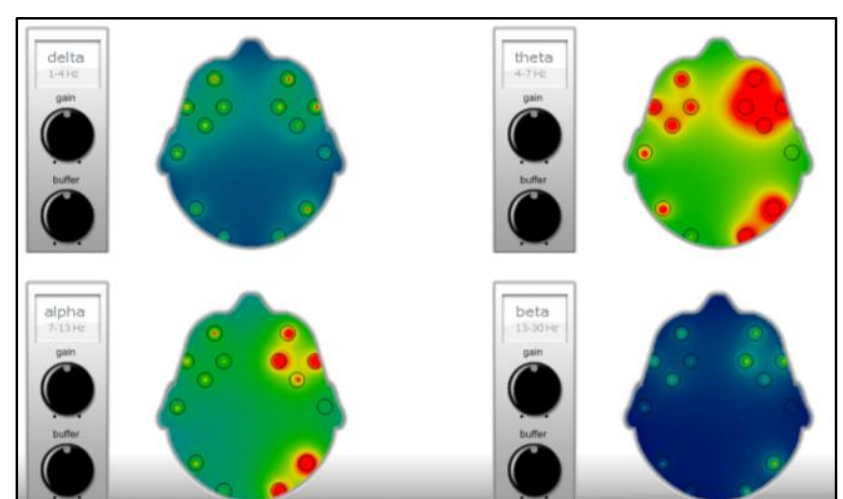

Fig. 10. Brain activity during the third type of listening relaxing music.

\section{Conclusion}

This paper briefly described what is Brain Computer Interface and its types. After that, electroencephalogram has been described. EEG is a non-invasive method can also be used for academic purposes. The measurement was mainly concerned with the frequency of brain waves. Brain waves, that occur during problem solving or relaxation, has been measured by the application Emotiv Brain Activity Map.

Our research deals with BCI system, which was identified by brain waves in two different actions. Firstly, we managed to measure data while the person solving a problem. Secondly, we measured the person while relaxing. There were used the type of relaxing music. After that, we evaluated and described the collected data. This measurement is the beginning of the further research. Future work lies primarily with the purchase of PRO license that will enable the raw EEG signal to be processed further. The RAW EEG signal can also be processed in other applications instead of Emotiv applications. This type of the measurement could be taken by people with epilepsy or other abnormalities in clinical EEG. BCI technology is a relatively new research area with great application potential. This is mainly a possible improvement in the quality of life for patients with permanent neurological deficits. By implementing this method into neuro-rehabilitation practice, we can improve the patient's health and mental state.

This work was supported by Internal Grant Agency of Tomas Bata University in Zlin under the project No. IGA/FAI/2018/008.

\section{References}

1. S. S. Mader, M. Windelspecht, Human biology, 12th ed., (New York, NY: McGraw-Hill, 2012), ISBN 0073525464

2. H.S. Anupama, N.K. Cauvery, G.M. Lingaraju, International Journal of Advances in Engineering \& Technology, 3, 739-745 (2012)

3. S. Xie, W. Meng, Biomechatronics in medical rehabilitation, (New York, NY: Springer Berlin Heidelberg, 2017), ISBN 9783319528830

4. B. HE, Neural engineering, Second edition. (New York: Springer, 2013)

5. S. Siuly, Y. Li, Y. Zhang, EEG signal analysis and classification, (New York, NY: Springer Berlin Heidelberg, 2017), ISBN 9783319476520.

6. 5 Types Of Brain Waves Frequencies: Gamma, Beta, Alpha, Theta, Delta [online], in: https://mentalhealthdaily.com/2014/04/15/5-typesof-brain-waves-frequencies-gamma-beta-alphatheta-delta/

7. Ch. S. Nam, A. Nijholt, F. Lotte, Brain-computer interfaces handbook: technological and theoretical advances, (Boca Raton: Taylor \& Francis, CRC Press, 2018), ISBN 9781498773430.

8. J. R. Wolpaw, E. Winter, Brain-computer interfaces: principles and practice, (New York: Oxford University Press, c2012), ISBN 9780195388855 .

9. D. Puthankattil Subha, P. K. Joseph, R. Acharya U, Ch. Min Lim, EEG Signal Analysis: A Survey, Springer Science 34, 195-212 (2007)

10. N. Thi, H. Hanh, H. Van Tuan, Identification of some brain waves signal and applications, ICIAE 12, 1415-1420 (2017) 